13. Ranke; Nederl. Tijdschr. v. Geneesekunde, I $88_{4}$, No. 5 I. Reussen ; Centralbl. für Chirurgie, I $S_{5} 5$, No I 5 , p. 280.

I4. Sordina; Rivista veneta di Scienze med., September, I 885. Centralbl. fuir Chururgie, IS's, No. 3, p. $4 \mathrm{~S}$.

15. Kappeler; Ueber grosse atypische Resectionen am Fusse. Deutsche Zeitschrift für Chirurgie, Bal. xxx, p. 432, ISSo.

I6. Conner; Excision of the Tarsus. Transactions of the American Surgical Association, vol. i, p. 285 .

17. Monastyrsky ; Zur Steuer der Wahrheit, etc. St. Peters. burger med. Wochenschrift, January I I (23), I $\$ 86$.

\section{THE ETIOLOGY AND CURE OF ASTHMA.}

Read before the Chicago Medical Society, Fanuary 3, 1887. BY EDWIN J. KUH, M.D.,

SURGEON TO THE MICHAEL REESE HOSPITAL, CHICACO.

The work of Wilhelm Hack on the radical treatment of migraine, asthma, hay fever and other neuroses, has received very inadequate recognition in this country. By writers on hay fever he is frequently quoted in an offhand manner together with a string of other authors; so that one derives the impression that few of those who quote him have read him. And if his specialistic colleagues do not do him justice, the large class of general practitioners ignore him almost altogether. His work is-taken altogetherof even greater interest to the physician than to the specialist, and it is a deplorable consequence of specialistic exclusiveness, that the results of his work have not yet received wider recognition among us. He teaches us that the rhinoscope must forthwith be as indispensable an instrument for all physicians as the thermometer and stethoscope.

The value of Hack's discovery, that asthma nervosum is a reflex disease with, usually, the nose as a starting-point, can best be appreciated by one who himself, for many years, struggled against the disease and fumed at the utter impotence of medical art to stave off the attacks. If $I$, therefore, in the course of this paper, class myself among my own patients, I shall do so with the view of bringing the subject within closer range. It is foreign to my subject to consider the isolated publications, from Voltolini downward, on the dependence of asthma upon polypous growths in the nose. Such cases are infrequent enough to be almost considered curiosities (Michel, for instance, reports 135 cases of polypus without asthma); and as Hack shows, polypi have rather a tendency to prevent asthma than to cause it. It will also simplify our subject, if we omit hay fever from our consideration.

The form of asthma of which I wish to treat exclusively is that perennial form which is more or less in dependent of the seasons, namely: asthma nervosum, or "Essentielles Asthma" of the Germans. Some persons never get beyond a slight hint of asthma. They will from time to time make a heaving, sighing motion, or complain of præcordial fulness with or without palpitation, or of sudden drowsiness, or dream heavily at night and complain of dulness, lassitude and headache in the morning. This latter condition has many gradations, the culmination of which is nightmare. In the future we must, therefore, learn to distinguish between an incubus of gastric and of respiratory origin. Other half-asthmatics complain only of a fleeting, leaden heaviness in the limbs amounting almost to pain; the same sensation of which so many true asthmatics complain after an asthmatic night. The typical asthma nervosum is known to us all as a neurosis occurring in paroxysms.

The patient may or may not feel an aura. He will generally, towards evening, or when he lies down, or awakes in the night, begin to wheeze. This wheezing may be associated with itching in the nose, or sneezing, or coughing; the attacks last an indefinite time, and generally end with the expectoration of a transparent glassy mucus. Such patients are often free from asthma during the day. Physical and chemical irritants, such as dust, sudden changes in temperature, the inhalation of certain gases, and a long series of idiosyncrasies which we find enumerated in textbooks, can induce an attack. But the recumbent position is the most uniform exciting cause of the single paroxysms. Such patients may be free from chronic bronchitis, chronic emphysema, heart-, kidney-, intestinal- and uterine disease: hence the term "Essentielles Asthma."

When we read authors whose contributions to the study of asthma antedate the last few years, we are struck by the uniformity with which they cling to a pet theory, each of which seems to give satisfaction to its upholder. It is merely an evasion to say that asthmatic paroxysms are induced by bronchial spasms, or by hyperæmia of the bronchial lining, or by the presence of Leyden's crystals, or by phrenic spasm, or by bulbar irritation, or by exudative bronchiolitis. For any one of these presumable causes would demand a first cause, in order to merit etiological dignity.

A true etiology of asthma had therefore to be dis. covered, and Hack did it in the following manner: He knew, of course, of the occasional rôle of nasal neoplasms. Schäffer and B. Fränkel had also indicated that the sensibility of the nasal lining could be so heightened through chronic catarrhal conditions, as to be a starting point for reflex disturbances. Then Hack found that be could experimentally produce glottis-spasm by touching the turbinated bodies of a sensitive individual with a probe. He then reasoned as follows: A nasal mucous membrane which shows merely slight affection, and which is not deadened in its sensibility by thickening and hypertrophy, is perhaps a better surface for exciting reflexes than one which shows evident signs of disease. And if this were the case, he reasoned, then perhaps the importance of nasal reflexes had been formerly overlooked just because of the insignificant abnormities of such a sensitive nose.

The very frequency of certain conditions may have given rise to an under-estimation of their significance. And so Hack systematically examined the nose of every patient who, for whatever ailing, came within his reach. He learned to make one distinction very rapidly, namely: that what is usually termed hypertrophic nasal catarrh is a twofold condition, which in its effects is quite opposite. In the anatomically true rhinitis hypertrophica the mucous membrane is really thickened, hypertrophied through chronic inflammation. Pressure with a probe meets with a certain 
unyielding resistance, and there is a purulent, crusty secretion. This form does not give rise to reflex disturbances.

But there is another form, a pseudo-hypertrophy, the importance of which it is Hack's merit to have pointed out. It is that transitory swelling of the cavernous tissue of the inferior and middle turbinated bodies, which has of late been so often described that I spare you a repetition. In this form the nose may either have a very dry, itchy sensation, or show copious watery secretion. Compression with a probe gives the air-pillow reaction. Sach individuals show fleeting alternate or synchronous obstruction of the nasal cavities. Often, when examining the nose of patients, we notice sudden engorgements and collapse, so that Hack's term erectility is not an exaggeration. These cavernous bodies with their frequently anæmic covering form a link in certain morbid reflexes, and when this link is destroyed through operative intervention, the reflexes cease. No symptom is more frequently overlooked by patients than transitory nasal obstruction. Most patients will positively deny its existence, until it is demonstrated to them. 'Therefore the assurance of an asthmatic that his nose has always appeared healthy is of no value.

Other asthmatics, if conscious of nasal trouble, consider it simply concomitant with their asthma, and it is characteristic of them that they will often resist the inquiries of the physician who attaches so muchi importance to rhinoscopic examination and nasal symptoms, when all their trouble seems located in the chest. It is interesting to observe how such people become gradually convinced, and how uniformly they marvel at having forgotten or overlooked most constant symptoms. Only recently I succeeded in showing an asthmatic half a dozen rapid openings and closings within barely more than a minute or two.

The theory of Hack is a simple one, and although it does not cover all the ground, is a very satisfactory one. He says that the turbinated bodies become engorged through various irritants, and that this vaso. dilatatory disturbance is transmitted to the bronchial tubes in asthma. The turbinated bodies act as accumulators for reflexes, store them up, as it were, and then transmit them to other parts. A destruction of the nasalswelling removes the reflexes. The experiences of numerous writers since 1883 corroborate the correctness of Hack's discovery.

By way of illustration I could not, I believe, select a better type of asthma of long standing than that of my own person. Twenty years ago, when I was 8 years of age, I became subject to so-called colds in the head and on the chest. They increased in severity and frequency from year to year, so that my surroundings were often puzzled to find an explanation for each outbreak. Presently nightly dyspnœa began to set in, in the following manner: During the day my respiration was quite free, but as soon as my head touched the pillow, the first wheeze set in; the paroxysms were very severe. They ceased, after lasting throughout the night, in the morning, with the usual expectoration of glassy mucus.

During the day there was never any difficulty, ex- cept when occasioned by laughter. I,aughter would infallibly cause itching under the chin and between the scapula, then I would cough convulsively and the attack was upon me. But the recumbent position was the surest exciting cause. During the first years I also suffered from that form of conjunctivitis which is now known to arise from nasal disorder.

I must give Dr. Abram Jacobi, of New York, under whose treatment I was at the time, credit for having already then, even without the use of the nasal speculum, laid stress upon a nasal trouble. But the aggravation of my troubles which followed upon the introduction of weak nitrate of silver solutions into my nose, made the memory of him a less pleasant one in those years than it is at present. The greater part of 1870 to $187.5 \mathrm{I}$ spent in the Swiss mountains, where I was entirely well. The attacks ceased from the day on which I reached the mountains, and infallibly returned on the very day I left them. Once during harvest season in Bavaria (1872), while I sat in a meadow, I was suddenly overtaken with convulsive sneezing, coughing and asthma. It lasted hours before I could reach the neighboring village. During that same period I developed a peculiar idiosyncrasy towards dinner. In the midst of the meal I would invariably for weeks be seized with a convulsive cough, so severe that it threw me to the ground. Asthma was never absent in these attacks. Then, at other times, one or two or three sneezes would initiate an asthmatic attack; or sometimes, especially after traveling, I would sneeze sixty or seventy times without intermission. In those years I had the sensation as if the asthma were brought on by a swelling, which seemed to begin above and behind the palate (it was associated with intense itching, which I attempted to relieve by rubbing my tongue against the hard palate), and traveled downward to the posterior pharynx, then seemed to skip the larynx and continued from the trachea downward. This phenomenon lasted a few seconds, and then the attack began. Railroad travel would invariably cause a night of asthma. One hotel, at which I was frequently obliged to stop in Germany, adjoined a stable, and was regularly the cause of some of the severest attacks.

The few years which antedated my acquaintance with Hack's writings were comparatively easy ones, because the inhalation of Kidder's asthma pastilles - the only palliative I ever used successfully-gave me very great relief. They not only immediately terminated an attack, but also prevented their occurrence for the next hours.

As soon as I became acquainted with Hack's articles in the Berliner klin. Wochenschrift, of 1882 , and with his monograph in 1883 , I commenced stricter self-observation, and found the following:

As soon as I lay down my nose would become obstructed. The occlusion corresponded to the side on which I lay. By turning over, the occluded side would open and the other close. To have any part of the nasal mucous membrane touched by a probe gave such intense pain that I could not suppress an outcry. I could bring on an attack of asthma by rubbing my ala nasi against the septum. 
Never did I feel the slightest dyspncea when nasal in respiration was free, and never was nasal respiration obstructed but what I felt asthmatic distress.

Under these circumstances there could be no hesitation; Dr. Jefferson Bettman (now of New York) and Dr. Henry Gradle, performed the galvano-caustic "destruction" of both inferior turbinated swellings. When I say galvano-caustic "destruction," I should like to put the word destruction in quotation marks; for I have found the radical obliteration of the entire inferior turbinated bodies almost an impossibility. Hack demands, and my experience confirms the correctness of his view, that the radical cure of asthma demands the radical destruction of the cavernous erection. But a longer and closer observation of such patients in whom the extirpation seems complete will almost invariably show relapses, which must again be subjected to operative interference.

In my own case fourteen cauterizations, performed with both a flat and furrow electrode in the manner described by Hack, have not succeeded in permanently clearing the nose. The asthmatic attacks have, to my unspeakable relief, ceased. Sleep is now a function of which I have lost all dread. But during the daily occurring fleeting occlusions, there is a feeling of heaviness on the chest and of excessive fatigue in the limbs, which do not pass away until the nose is free.

What is it that causes nasal occlusion? I have observed myself so closely in this regard, and have so many corroborative observations of intelligent patients, that I can make these positive statements:

Firstly, the fullness of the turbinated bodies is reg. ularly influenced by gravitation, and corresponds to the position of the head.

It is furthermore influenced by the temperature, and probably much more so by artificial warmth than cold; an over-heated room will almost invariably cause swelling in such patients. But the most dangerous and permanent cause of nasal obstruction is the inhalation of dust.

The time is, I hope, not far distant when our views on the etiology of respiratory diseases will undergo a radical change. The superstition of catching cold has lived too long. The light which mycological research has thrown on the etiology of most infective diseases must soon influence us toward a conviction that respiratory diseases are inhaled, not caught, and that suppuration in the respiratory tract is as impossible without the presence of micro-organisms, as it is on a wound. The superstition of "catching cold" is so pernicious because it diverts attention from the entrance-way of disease-generators. It is as impossible to contract an acute bronchitis through temperature influences alone, as it is to contract tuberculosis through a cold.

It is therefore of the utmost importance to warn asthmatics that as perfect an avoidance of dust in. halation as is possible in our contaminated surroundings is necessary to prevent a recurrence of their trouble. Not only the dust in the streets, but also that in our houses, is to be avoided as much as possible. Carpets and curtains are great receptacles of dust; and a strict regulation of street sprinkling will in the course of years, when the true etiology of res. piratory diseases will have been recognized, be considered as important a municipal regulation as the regulation of sewerage.

When are we to operate on asthmatics? The more recent the asthmatic trouble and the more pronounced the nasal symptoms, the better the prognosis. When complicated with chronic bronchitis and chronic emphysema, the outlook is generally bad. A most thorough examination of heart, lungs, kidneys and intestines should precede any operative interference. In cases of cardiac and nephritic asthma with nasal complications, I have never cauterized. Firstly, because it has seemed to me irrational; and secondly, because I feel so much gratitude towards Hack's discovery, that I shun any risk which might discredit it.

In some cases it is very difficult to decide whether an operation should be performed or not. For instance, in cases of long standing, say fifteen or twenty years, in which in the first years the nasal symptoms were very pronounced, but in later years have almost or entirely disappeared, in such cases cauterization is sometimes successful, but generally it is ursuccessful.

Cases in which the asthma is more or less constant and has lost its paroxysmal nature, give a doubtful prognosis. It has been a matter of experience with me, that those patients to whom the inhalation of Kidder's pastilles, or the application of cocaine to the nose (four per cent. solution on cotton), gives relief, afford a nuch better prognosis than others.

In asthmatics in which coughing precedes the attack and all nasal symptoms are missing, nasal cauterization will cure, if the cough is a so-called nasal cough.

There are a number of asthmatics, fortunately a minority, who seemingly offer a good prognosis, but with whom, for unknown reasons, the operation will fail. There can now be no doubt that there are other starting points for reflexes in the respiratory tract, besides the nose. The works of Trautmann and Tornwaldt have already added the vault of the pharynx to this list.

The bronchial tubes themselves can act as a starting point, as I can demonstrate on myself when I walk against a piercing wind, or inhale vapors of sulphurous acid with my nose plugged. So that, as Hack himself warningly says, we must not over-estimate the applicability of his discovery.

We must accuse the nose per exclusionem. Examine every patient thoroughly in every direction, and examine the rose last, is what I should like to advise.

About the operation itself, little is to be said. It is, as far as we know, absolutely harmless. I have performed many hundred cauterizations without any noteworthy complications. I have never had any traumatic infection. I insufflate iodoform or iodol upon the wound, introduce a pledget of cotton for a a few days, and keep my instruments aseptic.

The results are, on the whole, extremely gratifying. Asthma of many years' standing is sometimes broken after the very first cauterization. Almost all patients are relieved and many cured in the strict 
sense of the word. Some have relapses, which ad- easy and free from severe pain. I again examined ditional cauterization will remove. Others again the os and found it more soft and was enabled to inmay relapse with a new reflex sensitiveness in other sinuate my index finger, and by careful pressure with parts.

But on the whole the subject, still so new, still so my left hand over the fundus uteri I could distincapable of growth, broadening and development, is the true character of the pains I remained at the one of the most pleasing contributions to medical bedside; the pains were irregular, sometimes severe, knowledge, and the name of Hack will be, I am again they would be but slight. I examined the sure, not soon forgotten.

\section{A CASE OF ANTE-PARTUM HÆMORRHAGE AT TERM. RECOVERY.}

Read before the Chicago Medical Society, Fanuary 3, 1887. BY AUGUSTUS V. PARK, M.D.,

of chICAGo.

MeMBer of the american medical associaton, etc.

On the morning of August 5, 1886, I was called to see Mrs. S., age 37 years, a large and powerfullybuilt American-born Irish woman, cultured and intelligent for one in her station of life; she gave me this history:

'This was her ninth confinement, she had also suffered six miscarriages. Professor Daniel T. Nelson, her family physician, was compelled to use instruments on one occasion; the child was large and healthy and she made a good recovery; she had received no falls or injuries since carrying this child, to her knowledge. There was no history or evidence of specific disease. Before I finished questioning she remembered a few evenings since of running against some obstruction in the back-yard; this gave her a slight shock just at the time, but she paid no attention to it. The evening previous to my call, just as she had retired for the night, she said she was taken with a severe hæmorrhage which lasted for some time, and the amount of blood frightened her, and she called in a physician. The hæmorrhage soon stopped and her pains were nothing to speak of. There had been some propulsive efforts throughout the night, and in the morning they were simply teasing and prevented her from securing any rest. She suffered no real or hard labor pains and not a particle of hæmorrhage during the night.

I made a careful examination of the bedding and of the soiled clothing which had been removed the evening before, and found them wet and heavy and blood-stained, but was unable to find clots of arterial blood or any evidence of a severe or continued uterine hæmorrhage. The pulse was regular, the volume and strength good, respiration and temperature normal; vaginal examination revealed a rigid os uteri and situated high.in the pelvis, and directed backward so it pointed toward the cavity of the sacrum; the os uteri in this situation would have escaped any ordinary examination; there was no dilatation. was satisfied that the pains were not true labor pains and I gave tinctura opii deodarata in fifteen minim doses, and to repeat in an hour or two if necessary.

3 P.M.-I was sent for in great haste, and upon my arrival found that there had been expulsive efforts during the past hour, but she was again quite condition of the os uteri during the period of the pain, and found that the pains had no effect upon the cervix which remained undilated and flaccid, and the membranes did not become prominent or tense. The bowels had moved early in the day and a light meal taken at $12 \mathrm{M}$. Advised the continuance of the anodyne at once and again in two hours. This would control the severity of the pains and would lessen the rigidity of the os.

i I P.M.-Found the patient suffering true labor pains, pain in the back, the os soft and dilated to the size of a twenty-five cent piece; the head at the brim of the pelvis; the edges of the os thinned; the cervix rigid with each pain; there was no bag of water to act as a cushion. I 2, I and 2 o'clock the labor was much the same, and was what would be termed a tedious' labor; the liquor amnii having all escaped with the so-called hæmorrhage and each expulsive effort accomplished but little.

$2: 30$ A.M. - The pains are more regular, the patient is warm and perspiring; the face is flushed and the carotids stand out round and full with each parturient effort; everything seemed favorable for an early termination of labor. Soon I noticed that the pains were not as propulsive, yet they were equally as painful; complained of great thirst, constantly calling for water; she became uneasy and restless, the face losing its ruddy color and the lips bleached; the pulse feeble, rapid and easily compressible. For the first time in nearly four years of active obstetric practice I was brought to the full realization of the great danger of concealed or ante-partum hæmorrhage. There was but one thing to be thought of, and that was to deliver at once and in the short. est time practicable. I placed the patient across the bed, the head and shoulders without bolsters, the nates drawn forward over the edge of the bed, the knees well flexed and held by assistants. With the first pain I ruptured the membranes; this was followed with a gush of blood, a small amount however. I applied the forceps; the head was at the superior straight, and with the second pain I made gentle and careful traction, observing the well-known law which governs the obstetricians in the high forceps operation, and delivered a still.born child which bore all the evidences of having been dead at least six hours; gave child to the nurse and applied my left hand over the fundus uteri and made gentle pressure; soon the uterus commenced to contract and expel its contents; the blood and blood.clots that were thus forced out filled a common wash basin. The placenta was high up and normally situated on the posterior wall; with my left hand still on the fundus I experienced no trouble in reaching and removing the placenta; with this accomplished, all hæmorrhage ceased, ergot was given and gentle 